\title{
Introduction of sample tubes with sodium azide as a preservative for ethyl glucuronide in urine
}

\author{
Marc Luginbühl $^{1}$ - Wolfgang Weinmann ${ }^{1}$ - Ali Al-Ahmad ${ }^{2}$
}

Received: 27 December 2016 / Accepted: 4 July 2017

(C) Springer-Verlag GmbH Germany 2017

\begin{abstract}
Ethyl glucuronide (EtG) is a direct alcohol marker, which is widely used for clinical and forensic applications, mainly for abstinence control. However, the instability of EtG in urine against bacterial degradation or the postcollectional synthesis of EtG in contaminated samples may cause false interpretation of EtG results in urine samples. This study evaluates the potential of sodium azide in tubes used for urine collection to hinder degradation of ethyl glucuronide by bacterial metabolism taking place during growth of bacterial colonies. The tubes are part of a commercial oral fluid collection device. The sampling system was tested with different gram-positive and gram-negative bacterial species previously observed in urinary tract infections, such as Escherichia coli, Staphylococcus aureus, Enterecoccus faecalis, Staphylococcus epidermidis, Klebsiella pneumoniae, Enterobacter cloacae, and Pseudomonas aeruginosa. Inhibition of bacterial growth by sodium azide, resulting in lower numbers of colony forming units compared to control samples, was observed for all tested bacterial species. To test the prevention of EtG degradation by the predominant pathogen in urinary tract infection, sterile-filtered urine and deficient medium were spiked with EtG, and inoculated with E. coli prior to incubation for 4 days at $37^{\circ} \mathrm{C}$ in tubes with and without sodium azide. Samples were collected every 24 hours, during four consecutive days, whereby the colony
\end{abstract}

Wolfgang Weinmann

wolfgang.weinmann@irm.unibe.ch

1 Institute of Forensic Medicine, University of Bern, Bühlstrasse 20, 3012 Bern, Switzerland

2 Department of Operative Dentistry and Periodontology, University Medical Centre Freiburg, Hugstetterstrasse 55,

79106 Freiburg, Germany forming units (CFU) were counted on Columbia blood agar plates, and EtG was analyzed by LC-MS/MS. As expected, EtG degradation was observed when standard polypropylene tubes were used for the storage of contaminated samples. However, urine specimens collected in sodium azide tubes showed no or very limited bacterial growth and no EtG degradation. As a conclusion, sodium azide is useful to reduce bacterial growth of gram-negative and gram-positive bacteria. It inhibits the degradation of EtG by E. coli and can be used for the stabilization of EtG in urine samples.

Keywords Ethyl glucuronide $\cdot$ Microbial degradation · Preservative $\cdot$ Sodium azide $\cdot$ Preanalytical stability

\section{Introduction}

In order to prove alcohol consumption or abstinence, various direct alcohol markers are currently analyzed, such as ethyl glucuronide (EtG), ethyl sulfate (EtS), and phosphatidylethanol (PEth). EtG as a sensitive and specific short-term marker is thereby the most versatile, as it can be detected in urine, blood, and hair samples [1]. Bacterial degradation as well as neoformation of EtG from ethanol in urine samples containing bacteria have been reported [2,3]. Worldwide, no preservatives for stabilizing the analyte concentrations are used, although shipment of samples to a laboratory may take several days under non-cooled conditions. The preservation of urine samples was therefore extensively investigated $[4,5]$. However, the use of dried urine spots for inhibition of post-sampling EtG degradation is not popular until now, and the use of boric acid tubes turned out to be incompatible with the DRI® immunoassay for $\mathrm{EtG}$ detection [5, 6].

In this article, we investigate the preservation of sample integrity by using commercially available sodium azide tubes. 
Sodium azide is a well-established preservative against microorganisms, which generally inhibits microbial activity [7, 8]. Subsequently, it inhibits the growth of E. coli, the predominant pathogen (80\%) in urinary tract infections [9]. "Bacterial growth"-which is the increase of numbers of colony forming units (CFU) - within the sample would lead to degradation of $\mathrm{EtG}$, when EtG is used as a carbon source. Inhibition of major processes of the bacterial cell activity — as has been reported for sodium azide - leads to termination of substrate turnover (e.g., inhibition of DNA synthesis and cell division in Salmonella typhimurium by azide) $[10,11]$. As a consequence, the degradation of EtG would be stopped, when the bacterial cell activity stops. Our aim was to investigate, if sodium azide tubes can inhibit the bacterial growth of different gram-positive and gram-negative bacteria. Furthermore, investigation of sodium azide on the stability of EtG in urine inoculated with E. coli should be used to test the applicability of this stabilizing agent in analysis for EtG by LC-MS/MS.

\section{Materials and methods}

\section{Reagents}

Water was produced with a Milli-Q water system from Millipore (Billerica, USA). Acetonitrile (p.a.) was obtained from Acros Organics (Geel, Belgium). Isopropyl alcohol was purchased from Fisher Chemicals (Reinach, Switzerland). EtG was obtained from Medichem Diagnostica (Steinenbronn, Germany) and EtG- $d_{5}$ was obtained from Lipomed (Arlesheim, Switzerland). Formic acid (puriss p.a., 98\%) and methanol (spectrophotometric grade, $\geq 99 \%$ ) were purchased from Sigma-Aldrich (Buchs, Switzerland). Sodium azide tubes from the Saliva Collection System (SCS), containing $4 \mathrm{mg}$ of sodium azide per tube, were a gift from Greiner Bio-One International (Kermsmünster, Austria), see Fig. 1. Deficient medium glucose solution was obtained from the Institute of Medical Microbiology and Hygiene, Albert-Ludwigs-University, Freiburg. The deficient medium consisted of a $500 \mathrm{~mL}$ basic medium $\left(1.5 \mathrm{~g} / \mathrm{L} \mathrm{KH}_{2} \mathrm{PO}_{4}, 0.5 \mathrm{~g} / \mathrm{L} \mathrm{MgSO}_{4} \cdot 7 \mathrm{H}_{2} \mathrm{O}, 0.13 \mathrm{~g} /\right.$ $\mathrm{L} \mathrm{CaCl}_{2} \cdot \mathrm{H}_{2} \mathrm{O}, 5.0 \mathrm{~g} / \mathrm{L}\left(\mathrm{NH}_{4}\right)_{2} \mathrm{SO}_{4}$ and $\left.0.5 \mathrm{~g} / \mathrm{L} \mathrm{KNO}_{3}\right)$, $2 \mathrm{~mL}$ of a trace element solution $\left(5 \mathrm{mg} / \mathrm{mL} \mathrm{H}_{3} \mathrm{BO}_{3}\right.$, $0.4 \mathrm{mg} / \mathrm{mL} \mathrm{CuSO}_{4} \cdot 5 \mathrm{H}_{2} \mathrm{O}, 1 \mathrm{mg} / \mathrm{mL} \mathrm{KI}, 2 \mathrm{mg} / \mathrm{mL}$ $\mathrm{FeCl}_{3}, 3.6 \mathrm{mg} \mathrm{MnSO}_{4} \cdot \mathrm{H}_{2} \mathrm{O}$ and $7.1 \mathrm{mg} \mathrm{ZnSO} \mathrm{Zn}_{4} \cdot 7 \mathrm{H}_{2} \mathrm{O}$ ), and $10 \mathrm{~mL}$ of a $\mathrm{D}(+)$-glucose solution $(0.5 \mu \mathrm{g} / \mathrm{L})$. Additionally, $500 \mu \mathrm{L}$ of a growth substance solution was added $(2 \mu \mathrm{g} / \mathrm{mL} \mathrm{D}(+)$-biotin, $100 \mu \mathrm{g} / \mathrm{mL}$ Ca-pantothenate, $100 \mu \mathrm{g} / \mathrm{mL}$ p-aminobenzoate, $200 \mu \mathrm{g} / \mathrm{mL}$ thiamine). The rich medium consisted of tryptic soy broth (Merck, Darmstadt, Germany) including casein-peptone, soymealpeptone, and sodium chloride.

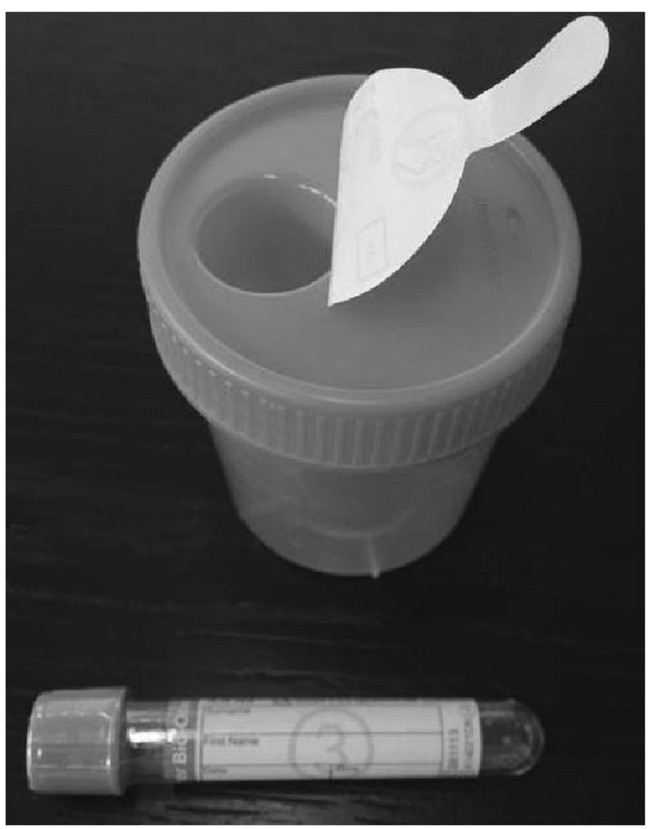

Fig. 1 Urine collection cup with sodium azide sample tube. The sodium azide tube can be filled by pushing the orange cap through the valve closure in the collection cup.The vacuum within the tube will automatically suck $2.8-3.5 \mathrm{~mL}$ of sample into the tube

\section{Study design bacterial growth study}

In addition to $E$. coli (2006/9478), a strain with $\beta$ glucuronidase activity which was previously isolated from autopsy material, the growth of the following gram-negative bacterial strains was tested with and without sodium azide: Pseudomonas aeruginosa ATCC 27853, Klebsiella pneumoniae IUK 1230, and Enterobacter cloacae IUK 611. Additionally, the growth inhibition by sodium azide of three representative grampositive bacterial strains was tested: Staphylococcus aureus ATCC 29523, Staphylococcus epidermidis DSM 1794, and Enterococcus faecalis T9. All tested gramnegative and gram-positive bacterial species are frequently associated with urinary tract infections. The bacterial growth of gram-negative bacteria was examined in the deficient medium for up to 2 days, whereas the growth of gram-positive bacteria was additionally tested in rich medium for 1 day due to their inability to grow in the deficient medium. Both culture media are described in the reagents section. The reduction of the bacterial growth caused by sodium azide was calculated in comparison to the bacterial growth in the negative control without sodium azide. The inoculation of the different culture media and the determination of the colony forming units (CFU) over the growth time were conducted in double, as described below in detail for E. coli (2006/9478). EtG degradation studies were performed only for E. coli. 


\section{Study design EtG in sodium azide tubes}

Different positive control samples (10 samples, in double), negative control samples (20 samples, in double), and experimental approach samples (10 samples, in double), with a volume of $3.5 \mathrm{~mL}$ each, were prepared and measured in a single measurement each, to investigate the degradation of EtG during 4 days, see Table 1. The urine used during the whole experiment was from an alcohol abstinent person, centrifuged at $5000 \mathrm{~g}$ for $10 \mathrm{~min}$ and subsequently sterile filtrated using Millex GP syringe driven filter units $(0.22 \mu \mathrm{m}$, Millipore, Carrigtwohill, Ireland). The deficient medium glucose solution was prepared by mixing $500 \mathrm{~mL}$ basic medium with $2 \mathrm{~mL}$ of the trace element solution, $10 \mathrm{ml}$ of the $\mathrm{D}(+)$-glucose solution and $500 \mu \mathrm{L}$ of the growth substance solution.

For spiking with the bacteria, an overnight culture of $E$. coli (2006/9478) was prepared at MC Farland $0.5\left(1.5 \times 10^{8} \mathrm{CFU} /\right.$ $\mathrm{mL}$ ), diluted by $1: 10$, whereby $10 \mu \mathrm{L}$ was spiked to each sample [12]. Samples containing EtG were spiked with a concentrated solution, resulting in a concentration of approximately $10 \mu \mathrm{g} / \mathrm{mL}$ each. Samples were incubated at $37^{\circ} \mathrm{C}$ (Thermo Scientific HERAcell 150i, Waltham, MA, USA) throughout the whole experiment. The colony forming units (CFU) were cultivated on Columbia blood agar (CBA) [13]. The CBA plates were incubated at $37{ }^{\circ} \mathrm{C}$ for 3 days under aerobic conditions. The CFU were counted using the Gel Doc EQ Universal Hood (Bio-Rad Life Science Group, Hercules, USA). EtG concentrations were measured by LCMS/MS. Samples were analyzed at day 0, 1, 2, 3, and 4. Liquid samples were directly frozen at about $-18{ }^{\circ} \mathrm{C}$ after sampling.

\section{Preparation and processing of samples for EtG analysis}

By adding $150 \mu \mathrm{L}$ of acetonitrile (containing the internal standard) to $50 \mu \mathrm{L}$ of diluted sample $(40 \mu \mathrm{L}$ of urine and
$10 \mu \mathrm{L}$ of water) in a $2 \mathrm{~mL}$ Eppendorf tube, proteins were precipitated. The samples were then vigorously shaken for 5 min on a VIBRAX VXR basic (IKA, Staufen, Germany). Afterward, the samples were centrifuged for $10 \mathrm{~min}$ at $16,000 \mathrm{~g}$ on a Mikro $220 \mathrm{R}$ (Hettich, Switzerland), before $100 \mu \mathrm{L}$ of the supernatant solution was transferred into a glass vial with insert $(0.35 \mathrm{~mL}$, Infochroma, Zug, Switzerland). The organic phase was evaporated to dryness at $50{ }^{\circ} \mathrm{C}$ under a gentle stream of nitrogen. The residue was dissolved in $100 \mu \mathrm{L}$ of reconstitution solution (water/acetonitrile, $95 / 5+0.1 \%$ formic acid, $(v / v)$ ). An aliquot of $10 \mu \mathrm{L}$ was injected into the LC-MS/MS system. A blank urine and a zero sample (from an abstinent person) with and without internal standard were included. After the measurement of the diluted sample, in order to remain within the calibration range, the sample concentration was back calculated to a non-diluted concentration. The chromatographic and mass spectrometric system and conditions employed for the analysis were described earlier with some modifications [4, 14-16]. The analytical method is based on an LC-MS/MS method on a 3200 QTrap instrument (Sciex, Toronto, Canada) with post-column addition of 2-propanol. Chromatographic separation was performed on a Synergi Polar RP column $(150 \times 2.0 \mathrm{~mm}, 4 \mu \mathrm{m})$ with a Polar RP precolumn $(4 \times 2.0 \mathrm{~mm}$, Phenomenex, Torrance, USA $)$. Samples were quantified based on a six point calibration from $0.1-10 \mu \mathrm{g} / \mathrm{mL}$. The LOD was established at $0.04 \mu \mathrm{g} /$ $\mathrm{mL}$ (signal to noise ratio of $3: 1$ ), the LLOQ at $0.1 \mu \mathrm{g} / \mathrm{mL}$. The method was fully validated at our institute and is currently used for routine analysis. By evaluating urine samples from six different subjects for selectivity and specificity, no significant interferences were observed (intra assay imprecision of $8.8 \% \mathrm{CV}$ and intra-assay accuracy of $99.5 \%$ ). By analyzing three series of six quality control samples at each concentration $(0.1,0.3,1,10 \mu \mathrm{g} / \mathrm{mL})$ on three different days, EtG showed a mean inter-assay precision of 4.9 $10.0 \% \mathrm{CV}$ and an inter-assay accuracy of 101.9-114.6\%.
Table 1 Positive and negative control samples and their composition

\begin{tabular}{llllll}
\hline Sample Name & Sample type & Medium & EtG $[\mu \mathrm{g} / \mathrm{mL}]$ & Bacteria & Type of tube \\
\hline A1 & Positive control & Deficient medium & 10 & E. coli & Untreated \\
B1 & Positive control & Urine & 10 & E. coli & Untreated \\
A2 & Negative control & Deficient medium & 0 & E. coli & Untreated \\
B2 & Negative control & Urine & 0 & E. coli & Untreated \\
A3 & Negative control & Deficient medium & 0 & E. coli & Sodium azide \\
B3 & Negative control & Urine & 0 & E. coli & Sodium azide \\
A4 & Experimental approach & Deficient medium & 10 & E. coli & Sodium azide \\
B4 & Experimental approach & Urine & 10 & E. coli & Sodium azide \\
A5 & Sterility control & Deficient medium & 0 & - & Untreated \\
B5 & Sterility control & Urine & 0 & - & Untreated \\
\hline
\end{tabular}


Fig. 2 Mean colony forming units (CFU) in percent of the initial value (day 0 ) measured after growth of different bacteria in the deficient medium with or without sodium azide. Each data point shown represents the mean $\mathrm{CFU}$ out of two measurements

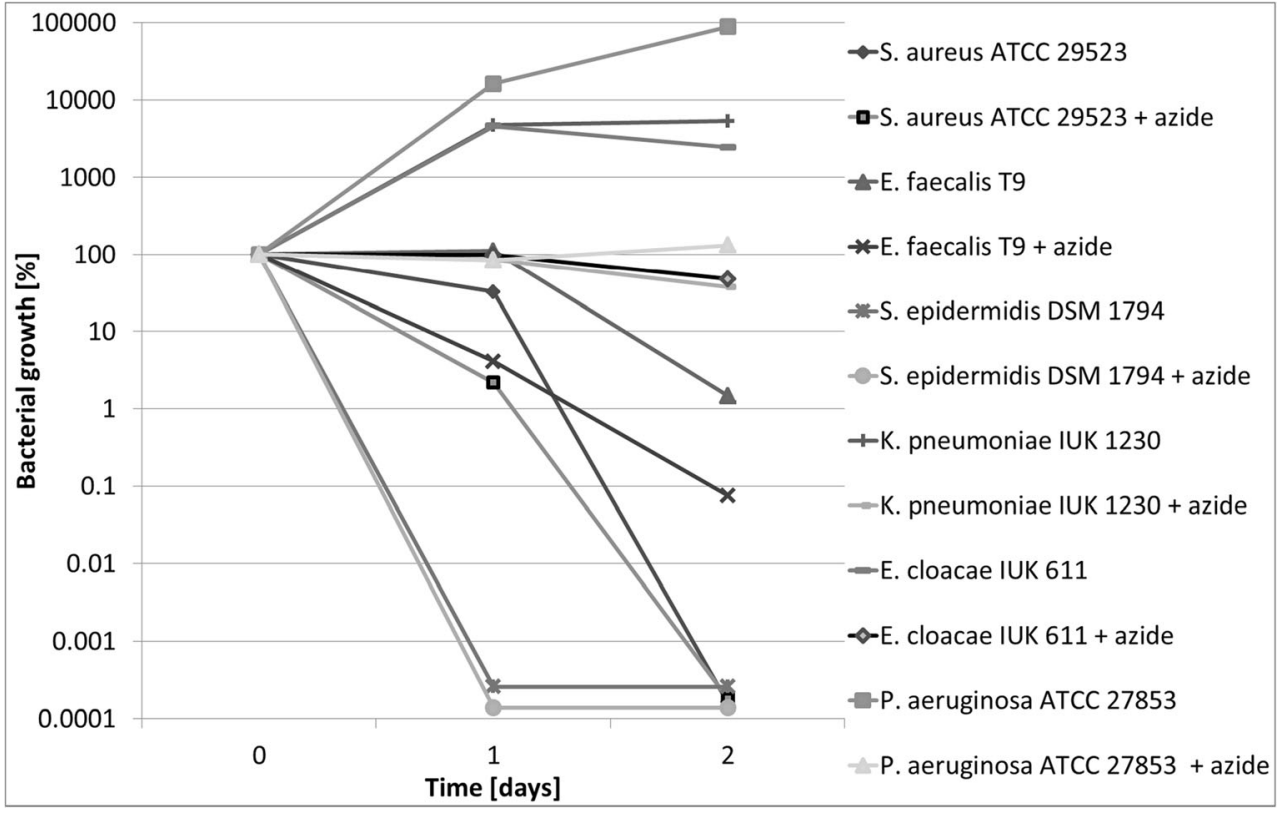

\section{Results}

\section{Bacterial growth study}

The growth of all tested bacterial strains in the deficient medium with or without sodium azide is depicted in Fig. 2. The growth of all gram-negative bacteria was inhibited by sodium azide compared to positive control samples in standard polypropylene tubes without sodium azide $(13 \mathrm{~mL}, 100 \times 16 \mathrm{~mm}$ $\varnothing$, Sarstedt, Nümbrecht, Germany). The CFU numbers of $K$. pneumonia and E. cloacae were 1-2 $\log 10$ higher than the initial value after growth for one or 2 days without sodium azide. This difference in the $\log 10 \mathrm{CFU}$ was even 2-3 for $P$. aeruginosa compared to the incubation with sodium azide. All gram-positive bacteria did not grow in the deficient medium. Nevertheless, sodium azide highly reduced the number of the CFU values of E. faecalis and $S$. aureus after 1 day of incubation in the deficient medium. The CFU values of S. epidermidis were reduced in the deficient medium even without sodium azide. According to these results, an inhibitory effect could only be observed for E. faecalis and S. aureus. To evaluate this, the antimicrobial effects of sodium azide against all three gram-positive bacterial strains were tested in rich medium. The growth of all gram-positive bacteria was
Fig. 3 Mean number of colony forming units (CFU) in percent of the initial value (day 0) measured after growth of the gram-positive bacteria in the rich medium with or without sodium azide. Each data point shown represents the mean number of CFU out of two measurements

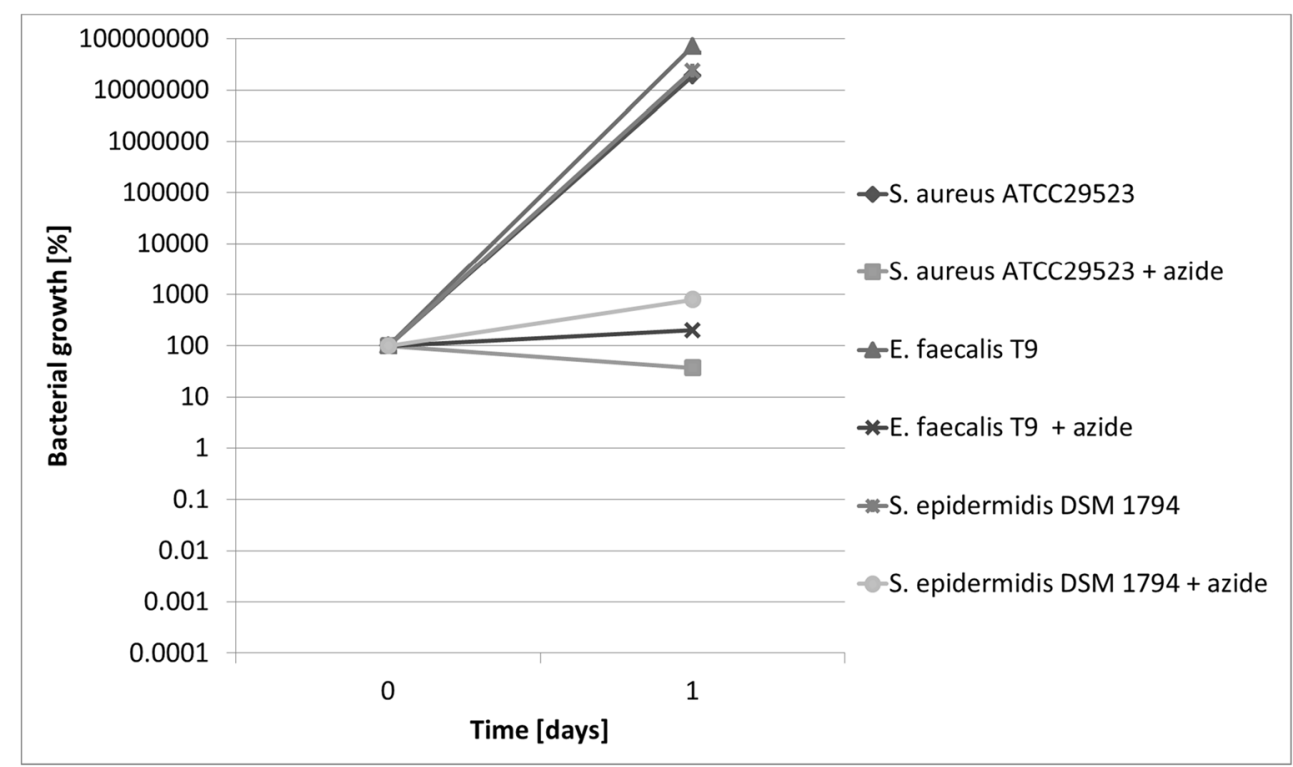


Fig. 4 EtG concentration changes over time for deficient medium samples. It is visible that samples stored in tubes without sodium azide (performed in double, $A 1.1$ and A1.2, see Table 1) show EtG degradation. Samples stored in sodium azide tubes (performed in double, $A 4.1$ and A4.2, see Table 1) show no EtG degradation. No EtG was measurable in negative control samples, not displayed here

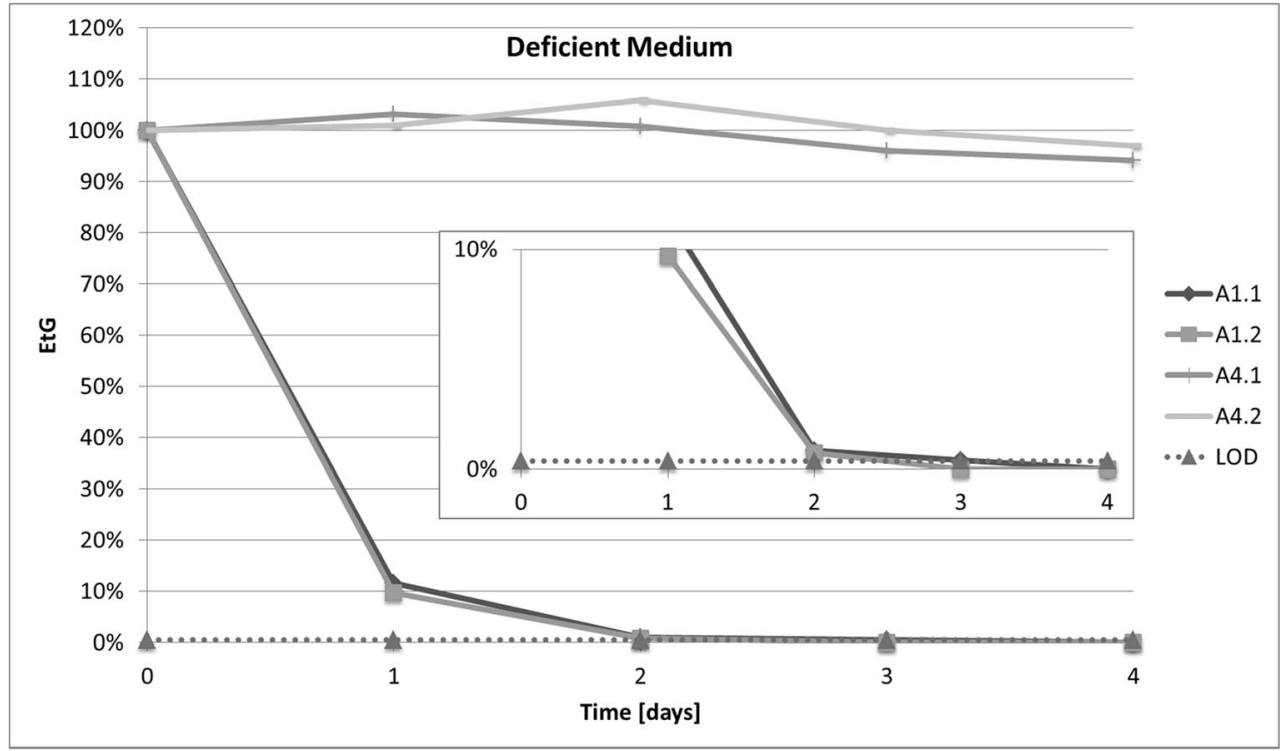

strongly reduced by sodium azide at a level of 5-6 $\log 10$ after 1 day of incubation when compared to positive control samples in standard tubes (see Fig. 3).

\section{EtG in sodium azide tubes}

EtG concentration changes for the two matrices, deficient medium and urine, are shown in Figs. 4 and 5, respectively. The mean number $(n=2)$ of the colony forming units (CFU) in the various samples are shown in Fig. 6. In agreement with literature reports, a degradation of EtG in both matrices was observed when bacteria were mixed and stored with EtG in untreated plastic tubes [4]. After the first day, about $90 \%$ of the initial EtG concentration had been metabolized by bacteria in deficient medium, compared to $100 \%$ degradation in urine after the first day. In deficient medium, EtG concentrations decreased to concentrations below LOD after 2 days of incubation. A clear increase in bacterial growth could be observed for both sample types. A closer look at the number of colony forming units in the different matrices revealed that the bacterial growth had been slower in deficient medium compared to urine. No degradation of EtG and no bacterial growth were observed when samples were frozen. Samples stored in sodium azide tubes showed no signs of EtG hydrolysis during the 4 days of measurement, as the quantification results remained within the confidence interval of $\pm 15 \%$ of the initial concentration [17]. Negative control samples, without any EtG, showed no signs of EtG formation. Sterility control of urine and deficient medium showed no increase in CFU and thus no signs of bacterial growth.

\section{Discussion}

Compared to standard polypropylene tubes for urine storage, suppressed bacterial growth was observed in all experiments,
Fig. 5 EtG concentration changes over time for urine samples. It is visible, that urine samples stored in tubes without sodium azide (perfomed in double, $B 1.1$ and B1.2, see Table 1) show EtG degradation. Urine samples stored in sodium azide tubes (performed in double, B4.1 and B4.2, see Table 1) show no EtG degradation. No EtG was measurable in negative control samples, not displayed here

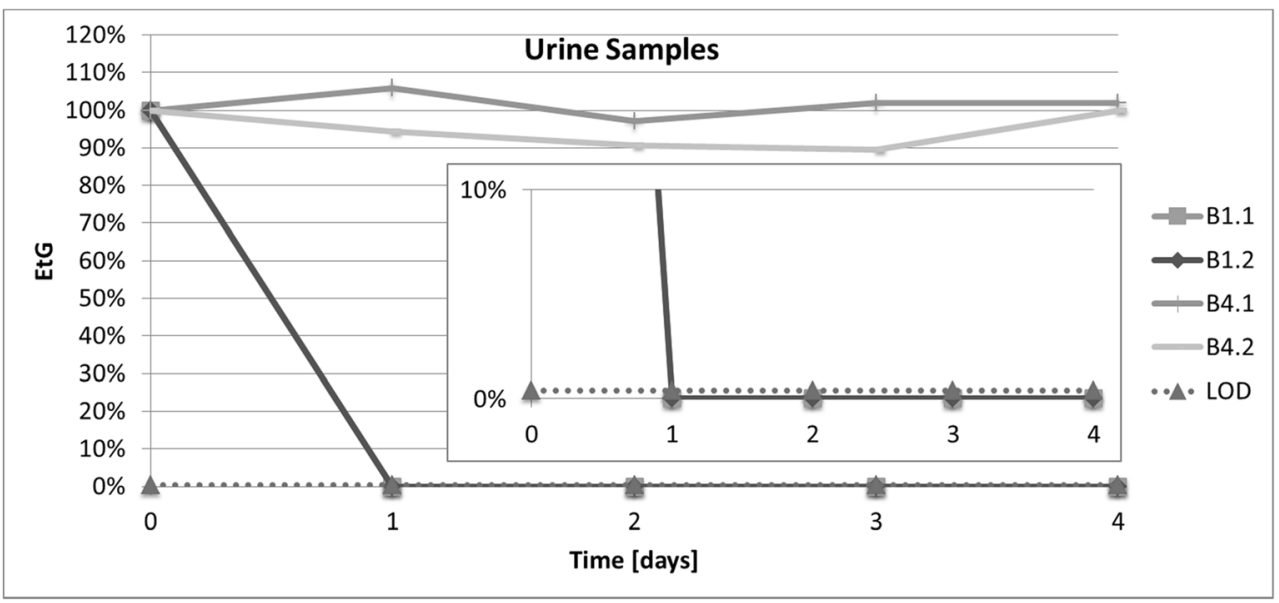


Fig. 6 Mean number of colony forming units measured during the time course of the experiment. Each data point shown represents the mean No. of CFU out of two measurements. In contrast to samples treated with sodium azide ( $A 3, B 3, A 4, B 4$, see Table 1), large increases in No. of CFU are visible for samples stored in tubes without sodium azide $(A 1, B 1, A 2, B 2$, see Table 1)

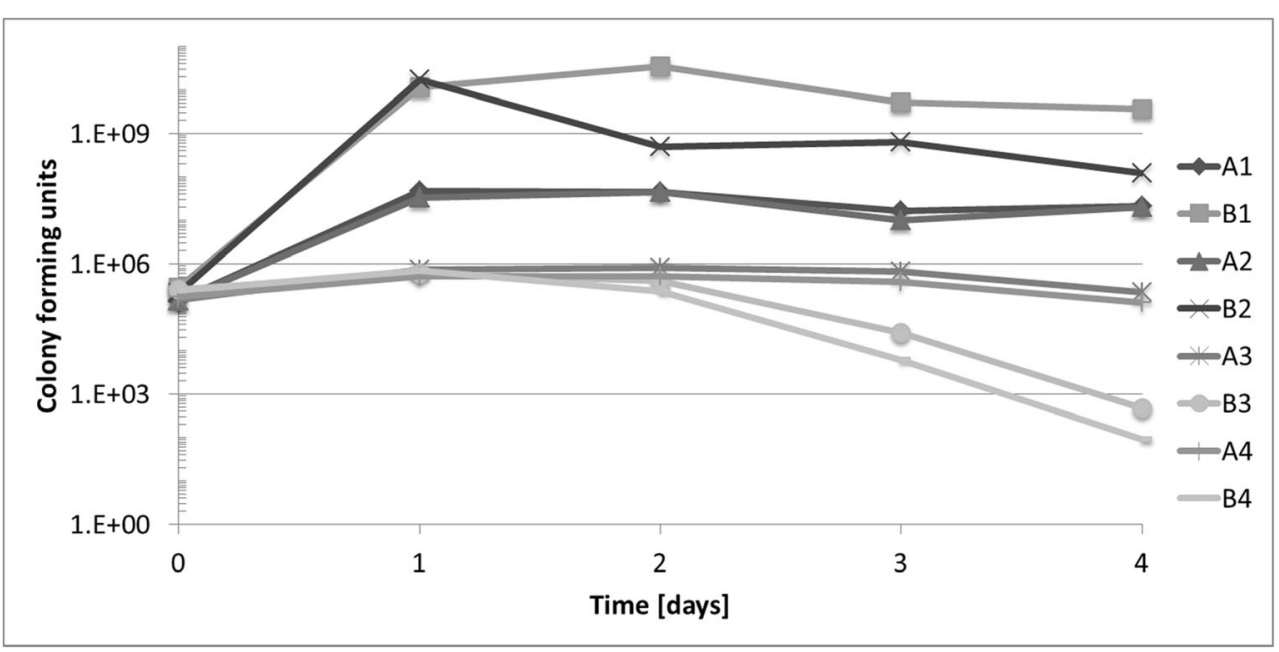

when sodium azide tubes were used. We could efficiently prevent the growth of bacteria of different species, gram-negative, as well as gram-positive species. The presented bacterial growth study showed that the composition of the medium plays a crucial role, when it comes to bacterial growth. Thereby, the investigated gram-positive bacteria proved to be demanding and did not grow in deficient medium. In the study of Helander et al., no EtG-degrading gram-positive bacteria were found in urine samples [9]. One reason might be that urine is not the appropriate medium for gram-positive bacteria due to the lack of essential nutrients, necessary for the higher demand of gram-positive bacteria to multiply. Baranowski et al. found gram-positive species which showed $\beta$-glucuronidase activity in autopsy material, such as Clostridium sordellii or Clostridium perfringens [3]. The use of sodium azide does not completely eliminate bacterial load within the sample. Nevertheless, bacterial activity and growth can be inhibited (bacteriostatic effects) by sodium azide, leading subsequently to inhibition of biological degradation of organic substrates in microbial niches. Such bacteriostatic effects have also been reported for some antibiotics which keep pathogen numbers under the infectious level [18]. Bacterial growth would lead to degradation of EtG, as it is used as a carbon source. Inhibition of major processes of the bacterial cell activity as has been reported for sodium azide, leads to termination of substrate turnover [10, 11]. In our EtG degradation experiment with $E$. coli, sodium azide successfully prevented bacterial growth and thus inhibited the degradation of EtG.

It should be mentioned, that in contrast to boric acid tubes, which have been tested in previous work, the sodium azide tubes from Greiner Bio-One (saliva collection system), used here for urine sampling, are compatible with the DRI® immunoassay for EtG (Thermo Fisher Scientific) - data not shown here $[5,6]$. In the unlikely case of EtG degradation within a sodium azide preserved tube, a quantification of EtS would remain the only option. Considering the toxicity of sodium azide as a chemical, it is important to mention, that the vacutainer-type collection tubes do not have to be opened for filling with urine, since they can be combined with the urine collection device from Greiner-Bio-One. This minimizes the potential contact with sodium azide by medical staff. For disposal, the sample tubes should be directed to waste combustion, since sodium azide decomposes at temperatures above $300{ }^{\circ} \mathrm{C}$.

\section{Conclusion}

Our study shows, that urine collection in sodium azide containing vacutainer-type tubes can prevent bacterial growth and thus prevent the degradation of EtG by bacteria during sample transportation and storage. Sodium azide proved to be reliable for the protection against preanalytical EtG degradation by E. coli, a gram-negative bacterium, which has been observed previously as a major source of bacterial contamination of urine specimens due to urinary tract infection. The growth of gram-negative and gram-positive bacteria, which is necessary for bacterial degradation of EtG, was effectively inhibited in different cultivation media upon use of sodium azide tubes, whereas in control tubes without sodium azide bacterial growth took place. By the use of sodium azide, degradation of EtG can be effectively hindered, even when samples are not cooled during transportation, thus eliminating the risk of having lower or even false-negative results for EtG. Based on our results, the utilization of sodium azide tubes enhances the reliability of EtG analysis for the detection of recent alcohol consumption.

Acknowledgements We would like to thank the Group for Oral Microbiology in Freiburg, Germany and the Team of Forensic Toxicology and Chemistry at the Institute of Forensic Medicine Bern, Switzerland. Furthermore, we would like to extend our gratitude to 
Michaela Brcak from Greiner Bio-One for providing the saliva transfer tubes from the saliva collection system.

Compliance with ethical standards Informend consent was given for obtaining the blank urine sample. All procedures performed were in accordance with the ethical standards of the institutional and national research committee and with the 1964 Helsinki declaration and its later amendments or comparable ethical standards.

Conflict of interest The authors declare that they have no conflict of interest.

\section{References}

1. Schröck A, Thierauf A, Wurst FM, Thon N, Weinmann W (2014) Progress in monitoring alcohol consumption and alcohol abuse by phosphatidylethanol. Bioanalysis 6(17):2285-2294

2. Helander A, Olsson I, Dahl H (2007) Postcollection synthesis of ethyl glucuronide by bacteria in urine may cause false identification of alcohol consumption. Clin Chem 53(10):1855-1857

3. Baranowski S, Serr A, Thierauf A, Weinmann W, Grosse Perdekamp M, Wurst FM et al (2008) In vitro study of bacterial degradation of ethyl glucuronide and ethyl sulphate. Int J Legal Med 122(5):389-393

4. Redondo AH, Korber C, Konig S, Langin A, Al-Ahmad A, Weinmann W (2012) Inhibition of bacterial degradation of EtG by collection as dried urine spots (DUS). Anal Bioanal Chem 402(7):2417-2424

5. Thierauf A, Serr A, Halter CC, Al-Ahmad A, Rana S, Weinmann W (2008) Influence of preservatives on the stability of ethyl glucuronide and ethyl sulphate in urine. Forensic Sci Int 182(1-3):41-45

6. Leickly E, McDonell MG, Vilardaga R, Angelo FA, Lowe JM, McPherson S et al (2015) High levels of agreement between clinic-based ethyl glucuronide (EtG) immunoassays and laboratory-based mass spectrometry. Am J Drug Alcohol Abuse 41(3):246-250
7. Packer RA (1943) The use of sodium azide $(\mathrm{NaN}(3))$ and crystal violet in a selective medium for streptococci and erysipelothrix rhusiopathiae. J Bacteriol 46(4):343-349

8. Lichstein HC, Soule MH (1944) Studies of the effect of sodium azide on microbic growth and respiration: I. The action of sodium azide on microbic growth. J Bacteriol 47(3):221-230

9. Helander A, Dahl H (2005) Urinary tract infection: a risk factor for false-negative urinary ethyl glucuronide but not ethyl sulfate in the detection of recent alcohol consumption. Clin Chem 51(9):17281730

10. Walton L, Elwell LP (1980) A microbiological assay for sodium azide. Anal Biochem 101(1):39-43

11. Ciesla Z, Mardarowicz K, Klopotowski T (1974) Inhibition of DNA synthesis and cell division in salmonella typhimurium by azide. Mol Gen Genet 135(4):339-348

12. Mc Farland J (1907) The nephelometer:an instrument for estimating the number of bacteria in suspensions used for calculating the opsonic index and for vaccines. J Am Med Assoc XLIX(14):11761178

13. Al-Ahmad A, Wiedmann-Al-Ahmad M, Auschill TM, Follo M, Braun G, Hellwig E et al (2008) Effects of commonly used food preservatives on biofilm formation of Streptococcus mutans in vitro. Arch Oral Biol 53(8):765-772

14. Hernández Redondo A, Schroeck A, Kneubuehl B, Weinmann W (2013) Determination of ethyl glucuronide and ethyl sulfate from dried blood spots. Int J Legal Med 127(4):769-775

15. Dresen S, Weinmann W, Wurst FM (2004) Forensic confirmatory analysis of ethyl sulfate - a new marker for alcohol consumptionby liquid-chromatography/electrospray ionization/tandem mass spectrometry. J Am Soc Mass Spectrom 15(11):1644-1648

16. Halter CC, Dresen S, Auwaerter V, Wurst FM, Weinmann W (2008) Kinetics in serum and urinary excretion of ethyl sulfate and ethyl glucuronide after medium dose ethanol intake. Int $\mathrm{J}$ Legal Med 122(2):123-128

17. FDA UDoHaHS (2001) Guidance for industry: bioanalytical method validation

18. Schnappinger D, Hillen W (1996) Tetracyclines: antibiotic action, uptake, and resistance mechanisms. Arch Microbiol 165(6):359369 